\title{
Multi-Criteria Decision Support Methods for Renewable Energy Systems on Islands
}

\author{
C. Wimmler, G. Hejazi, E. de Oliveira Fernandes, C. Moreira, and S. Connors
}

\begin{abstract}
Islands often are confronted with severe energy challenges especially those far from the main land which operate as isolated energy systems. In those cases electricity, the queen of the energy vectors shall be obtained from diversified sources to alleviate the burden of the dependence on fossil fuels. Thereby, the generation of electricity from renewable energy sources in combination with electricity storage becomes an irrecusably challenge in the nearby future. Often a variety of criteria can be applied to identify the suitability of technologies, whereas no ideal family of criteria has been defined in the literature. Hence, decision support for energy planning and management is required. This paper reviews the state-of-the-art of multi-criteria decision support methods applied to renewable energy and storage technologies. It will be analyzed where the current focus is placed on. The gaps of those analyzed studies will be evaluated and key aspects for future energy planning considerations for islands will be proposed. Finally, an outlook for a newly developed concept for island energy planning will be presented. Indeed, for most isolated islands renewable energy technologies in combination with storage devices are a desirable and valued solution for sustainable development.
\end{abstract}

Index Terms - Island energy planning, multi-criteria decision support methods, renewable energy technologies, sustainable development.

\section{INTRODUCTION}

Islands face diverse similarities in terms of their energy problems. Apart from their insularity and often not being grid-connected to the mainland energy network there are commonly social issues caused by not having sufficient energy, i.e. a high degree of young people unemployment that causes a trend of immigration, a lack of specialized work force, different levels of population density or a conservative mentality [1]. Plus, often welfare of islands depends on specific industries, mainly tourism, fishing and local businesses [2]. Even though tourism generates financial benefits, it is also the main driver for another problem; that of an increasing and highly season-depended energy and water demand [1], [3], [4]. Indeed, during the peak summer season many islands are confronted with energy security issues [5]. In correlation with tourism a high variation in the number of island inhabitants occurs [4], which during the main season might exceed that of the off-peak season by a few times [6].

Manuscript received February 15, 2014; revised June 24, 2014

C. Wimmler and G. Hejazi are with the Faculty of Engineering, University of Porto, Porto, 4200-46 Portugal (e-mail: c.wimmler@fe.up.pt golnar.hejazi@fe.up.pt).

E. de Oliveira Fernandes is with the Mechanical Engineering Department, University of Porto, Porto, 4200-446 Portugal (e-mail: eof@fe.up.pt).

C. Moreira is with the Electrical Engineering Department, University of Porto, Porto, 4200-446 Portugal (e-mail: clm@fe.up.pt).

$\mathrm{S}$. Connors is with the Massachusetts Institute of Technology, Cambridge, MA 02139-4307, USA (email: connorsr@mit.edu).
Considering that the energy demand will continue growing steadily in the next decades, precise energy planning and management as well as changing energy supply alternatives to more local ones can be a solution to overcome these issues [3].

Electricity represents the most flexible form of energy, as it can be used for heating, transportation and, of cause, as electricity. While fossil fuel imports are severely expensive and make an island dependent [7], the usage of locally available and abundant resources should be encouraged. In fact, most islands possess a diversity of resources, both on land and at sea. It is a matter of using these resources to foster sustainable development within an island energy system [3], [8], [9].

\section{SUSTAINABILITY ASSESSMENT}

Sustainability is certainly one of the major aspects for the realization of nearly any renewable energy project. Though, the indicators of a sustainability assessment need to be determined based on achieving a specific objective. As the solution should fulfill an islands energy requirements there are many real world factors that can be affected by the solution. Hence, it is essential to consider the criteria that can be relevant for renewable energy and storage technologies on islands. Since evaluating the consequences of the solution considering multi-criteria aspects is challenging, multi-criteria decision making (MCDM) methods can be applied to the energy planning problems. Such decision support is particularly valued by decision and policy makers.

Ness et al. [10] define "The purpose of sustainability assessment is to provide decision-makers with an evaluation of global to local integrated nature-society systems in short and long term perspectives in order to assist them to determine which actions should or should not be taken in an attempt to make society sustainable”.

A large variety of sustainable development indicators and sustainability assessment methodologies is presented in [11]. Thereby, an overall of 41 indices were identified and classified according to number of sub-indicators, scaling/normalization, weighting and aggregation. Besides efforts to measure sustainability as an integral approach, most cases only consider either environmental or economic or social aspects.

In the reviewed researches various sustainable indicators have been applied to renewable energy technologies (RETs) (Table II). The success of the assessment depends on the effectiveness of indicators for each criteria on that can correspond to a problem and/or fulfill the objective.

In [12] sustainability criteria (resource, environmental, economic and social) were used for technology selection. The 
considered indicators were efficiency $(\%)$, installation cost (USD/kW), electricity cost (ct/kWh), $\mathrm{CO}_{2}\left(\mathrm{kgCO}_{2} / \mathrm{kWh}\right)$ and area $\left(\mathrm{km}^{2} / \mathrm{kW}\right)$.

Another approach which only considers techno-economic criteria for onshore and offshore wind, geothermal, small hydropower, solar and photovoltaic power was presented by [13]. While the technical indicators are construction period, technical lifetime, capacity factor and maximum availability, the economic indicators are investment cost, fixed and variable operations and maintenance cost as well as progress ratio. An even more comprehensive list of indicators is presented in [14], whereas the sustainability aspects are grouped in technical, economic, environmental and social criteria (see Table I).

TABLE I: SELECTION CRITERIA FOR SUSTAINABLE ENERGy PLANNING [14]

\begin{tabular}{|l|l|}
\hline Triteria & Indicators \\
Technical & $\begin{array}{l}\text { Efficiency, exergy efficiency, primary energy ratio, } \\
\text { safety, reliability, maturity, others }\end{array}$ \\
\hline Economic & $\begin{array}{l}\text { Investment cost, operation and maintenance cost, fuel } \\
\text { cost, electric cost, net present value, payback period, } \\
\text { service life, equivalent annual cost, others }\end{array}$ \\
\hline Social & $\begin{array}{l}\mathrm{NO}_{\mathrm{x}} \text { emission, } \mathrm{CO}_{2} \text { emission, } \mathrm{CO} \text { emission, } \mathrm{SO}_{2} \\
\text { emission, particles emission, non-methane volatile } \\
\text { organic compounds, land use, noise, others }\end{array}$ \\
\hline $\begin{array}{l}\text { Social acceptability, job creation, social benefits, } \\
\text { others }\end{array}$ \\
\hline
\end{tabular}

Because of the nature of the systems the selection process for storage technologies is also based on different criteria and indicators. Barin et al. divide in qualitative and quantitative characteristics to determine the storage energy technology in a power quality scenario [15]. Qualitative indicators focus on load management, technical maturity and power quality; with the respective characteristics being load leveling, load following, spinning reserve, back-up or typical usage.

A sustainability index approach was undertaken by [16]. By this means, a weighted sum approach was used to quantify each indicator according to its importance. Criteria ranged from economic and environmental aspects to risk, but also considered indicators such as reliability, system life or energy density ratio. The evaluation reviewed lead acid and lithium batteries as well as fuel cells, whereas fuel cells come top in the selection process. Further selection procedures that considered similar selection criteria and indicators as the above mentioned researches are presented in [17]-[19].

This section of the paper clearly demonstrated that there are some common criteria and indicators. Though, it is essential to select an appropriate set of criteria based on the objective of the planning. The whole assessment should be considered as integrated process rather than a solitaire one. Plus, most indicators used for island cases are actually based on cases from the mainland. Therefore, it is necessary to consider criteria that specifically correspond to islands.

\section{Multi-CRITERIA DECISION MAKING MethODS FOR RENEWABLE ENERGY SYSTEMS}

MCDM can be an alternative to support decision and policy makers in their decision process. Depending on the values and preferences of a decision maker, different approaches can be attained. The selection process of an appropriate MCDM method for renewable energy planning is discussed in [20]. According to [21] the aims of MCDM are:

- "to aid decision-makers to be consistent with fixed 'general' objectives;

- to use representative data and transparent assessment procedures; and

- to help the accomplishment of decisional processes, focusing on increasing its efficiency."

A very comprehensive review of MCDM methods was undertaken by [22]. The analyzed methods include weighted sum/product method, analytical hierarchy process (AHP), preference ranking organization method for enrichment evaluation (PROMETHEE), elimination and choice translating reality (ELECTRE), technique for order preference by similarity to ideal solutions (TOPSIS), compromise programming (CP) and multi-attribute utility theory (MAUT). Additionally, Wang et al. [14] present fuzzy set methodology, grey relational method and others (i.e. Preference assessment by imprecise ratio statements (PARIS)) as MCDM methods for sustainable energy decision making. Besides, an integrated approach considering MCDM methods along with Geographic Information System (GIS) tools has been established by [23]. Indeed, combinations of GIS and MCDM are used for site selection of technologies; i.e. wind [24], solar [25] or tidal stream [26].

It should be noted that the main focus of this paper is not to analyze each method, its strengths and weaknesses, rather than to highlight the multitude of methods available. In the end, the method should be capable of incorporating different decision maker's preferences and data availability.

\section{MCDM METHODS, CRITERIA AND INDICATORS FOR SUSTAINABLE ENERGY PLANNING}

Extensive research in the permanent literature has been undertaken to assess the current state-of-the-art of applied and conceptual research in the area of MCDM for renewable energy and storage systems. Therefore, the purpose, criteria, indicators and MCDM method for each research were analyzed. Plus, the data type of criteria (quantitative, qualitative or mixed) and an evaluation if offshore and storage technologies were included in these cases were studied (see Table II). Additionally, Table III summarizes the applied system size and location as well as the considered technologies.

TABle II: Evaluation of Multi-Criteria Decision Making Methods ApPlied to Renewable Energy and Storage TeChNOLOGies

\begin{tabular}{|c|c|c|c|c|c|c|}
\hline & Purpose/target & Criteria and Indicators & $\frac{0}{\frac{0}{0}}$ & 帝 & \# & $\begin{array}{l}\text { Multi-criteria } \\
\text { decision making } \\
\text { method* }\end{array}$ \\
\hline \multicolumn{7}{|c|}{ Category: Impact assessment } \\
\hline Hong et al. [27] & $\begin{array}{l}\text { Impact of rural } \\
\text { electrification using RET to } \\
\text { improve energy access }\end{array}$ & $\begin{array}{l}\text { T - Ec: cost, life, power consumed, alternatives consumed } \\
\text { S - Ec: education level, occupation }\end{array}$ & $\mathrm{N}$ & $\mathrm{N}$ & $\begin{array}{c}6 \\
M\end{array}$ & $\begin{array}{l}\text { Multiple } \\
\text { correspondence } \\
\text { analysis }\end{array}$ \\
\hline
\end{tabular}




\begin{tabular}{|c|c|c|c|c|c|c|}
\hline \multicolumn{7}{|c|}{ TABLE II continued } \\
\hline Silva et al. [28] & $\begin{array}{l}\text { Assessment of rural } \\
\text { electrification with } \\
\text { renewable energy systems }\end{array}$ & $\begin{array}{l}\text { Ec: electricity generation cost } \\
\text { S: employment generation } \\
\text { En: land use, avoided } \mathrm{CO}_{2} \text { emissions }\end{array}$ & $\mathrm{N}$ & $\mathrm{N}$ & $\begin{array}{c}4 \\
\mathrm{QL}\end{array}$ & $\begin{array}{l}\text { Multi-objective } \\
\text { decision making- } \\
\text { goal programming }\end{array}$ \\
\hline $\begin{array}{l}\text { Cherni et al. } \\
\quad[29]\end{array}$ & $\begin{array}{l}\text { Calculate set of appropriate } \\
\text { energy options to fulfill local } \\
\text { needs }\end{array}$ & Physical, Financial, Natural, Social, Human & $\mathrm{N}$ & $\mathrm{N}$ & $\begin{array}{c}5 \\
\mathrm{QL}\end{array}$ & SURE tool \\
\hline Heo et al. [30] & $\begin{array}{l}\text { Establish criteria for } \\
\text { renewable } \\
\text { energy dissemination } \\
\text { programs }\end{array}$ & $\begin{array}{l}\text { T: Superiority of technology, Completeness of technology, } \\
\text { Reliability of technology and operation, Possibility of } \\
\text { acquiring original technology } \\
\text { Market: Domestic market size and competitiveness, Global } \\
\text { market size and competitiveness, Competitive power of } \\
\text { domestic technology } \\
\text { Ec: Supply capability, Economic feasibility, Supply } \\
\text { durability } \\
\text { En: Reduction of greenhouse gas and pollutants, } \\
\text { Requirement of resources, Acceptability of local residents } \\
\text { Policy: Contribution to achieve dissemination goal, } \\
\text { Spillover effect, Linkage with R\&D program, Influence of } \\
\text { existing social system }\end{array}$ & $\mathrm{N}$ & $\mathrm{N}$ & $\begin{array}{l}17 \\
\mathrm{QL}\end{array}$ & Fuzzy AHP \\
\hline $\begin{array}{l}\text { Chatzi- } \\
\text { mouratidis et al } \\
\text { [31] }\end{array}$ & $\begin{array}{l}\text { Evaluate impact on the } \\
\text { living standard of local } \\
\text { communities }\end{array}$ & $\begin{array}{l}\text { Quality of life: accident fatalities, non-radioactive } \\
\text { emissions, radioactivity, land requirement } \\
\text { S - Ec: job creation, compensation rates, social acceptance }\end{array}$ & $\mathrm{N}$ & $\mathrm{N}$ & $\begin{array}{l}7 \\
M\end{array}$ & AHP \\
\hline $\begin{array}{l}\text { Heano, et al } \\
{[32]}\end{array}$ & $\begin{array}{l}\text { Selecting energy generation } \\
\text { systems for improvement of } \\
\text { rural livelihoods }\end{array}$ & Physical, Financial, Natural, Social, Human & $\mathrm{N}$ & $\mathrm{N}$ & $\begin{array}{c}5 \\
\mathrm{QL}\end{array}$ & SURE tool \\
\hline \multicolumn{7}{|c|}{ Category: Power generation optimization } \\
\hline Stein [33] & $\begin{array}{l}\text { Rank various renewable and } \\
\text { non-renewable electricity } \\
\text { generation technologies }\end{array}$ & $\begin{array}{l}\text { Ec: total overnight cost, variable O\&M, fixed O\&M, fuel } \\
\text { cost; } \\
\text { T: Production efficiency, capacity factor } \\
\text { En: External costs, loss of life expectancy } \\
\text { S: fuel reserve years, job creation, net import as \% of } \\
\text { consumption }\end{array}$ & $\mathrm{N}$ & $\mathrm{N}$ & $\begin{array}{l}11 \\
\text { QN }\end{array}$ & AHP \\
\hline $\begin{array}{l}\text { Ribeiro et al. } \\
\text { [34] }\end{array}$ & $\begin{array}{l}\text { Support the evaluation of } \\
\text { different electricity } \\
\text { generation scenarios }\end{array}$ & $\begin{array}{l}\text { T: national industry, energy dependency, diversity of mix, } \\
\text { rate of dispatchable power } \\
\text { Ec: costs, investment in transmission network } \\
\text { En: visual impact, } \mathrm{CO}_{2} \text { emissions, land use } \\
\text { S: employment, local income, public health, noise }\end{array}$ & $\mathrm{N}$ & $\mathrm{N}$ & $\begin{array}{l}13 \\
\mathrm{M}\end{array}$ & $\begin{array}{l}\text { Value } \\
\text { measurement } \\
\text { method; includes } \\
\text { impact evaluation, } \\
\text { direct weighting } \\
\text { and trade-off } \\
\text { analysis }\end{array}$ \\
\hline Begic et al. [35] & $\begin{array}{l}\text { Multi-criteria sustainability } \\
\text { assessment of various } \\
\text { options of the energy power } \\
\text { system }\end{array}$ & $\begin{array}{l}\text { Resource: fuel, carbon steel, stainless steel, copper, } \\
\text { aluminum, insulation } \\
\text { En: } \mathrm{CO}_{2}, \mathrm{SO}_{2}, \mathrm{NO}_{\mathbf{x}} \\
\text { Ec: Energy cost, investment, efficiency } \\
\text { S: job, diversity }\end{array}$ & $\mathrm{N}$ & $\mathrm{N}$ & $\begin{array}{l}14 \\
\mathrm{QN}\end{array}$ & $\begin{array}{l}\text { Analysis and } \\
\text { synthesis of } \\
\text { indexes under } \\
\text { deficiency of } \\
\text { information } \\
\text { (ASPID) }\end{array}$ \\
\hline Suo et al. [36] & $\begin{array}{l}\text { Select optimal alternative } \\
\text { according to their optimism } \\
\text { degrees }\end{array}$ & $\begin{array}{l}\text { T: energy intensity, retirement, current capacity, potential } \\
\text { capacity, service life } \\
\text { Ec: O\&M cost, capital cost } \\
\text { En: GHG intensity }\end{array}$ & $\mathrm{N}$ & $\mathrm{N}$ & $\begin{array}{c}8 \\
\mathrm{QN}\end{array}$ & $\begin{array}{l}\text { advanced ordered } \\
\text { weighted } \\
\text { averaging } \\
\text { (AOWA) }\end{array}$ \\
\hline $\begin{array}{l}\text { Papadopoulos } \\
\text { et al. [37] }\end{array}$ & $\begin{array}{l}\text { Optimization of } \\
\text { decentralized/ isolated } \\
\text { energy systems }\end{array}$ & $\begin{array}{l}\text { Ec: NPV, life cycle cost, depreciated payback period, } \\
\text { black-out cost } \\
\text { En: } \mathrm{CO}_{2}\end{array}$ & $\mathrm{~N}$ & $\mathrm{~N}$ & $\begin{array}{c}5 \\
\text { QN }\end{array}$ & ELECTRE III \\
\hline $\begin{array}{c}\text { La Rovere et al. } \\
{[38]}\end{array}$ & $\begin{array}{l}\text { Analyze the sustainability of } \\
\text { the expansion of electricity } \\
\text { generation }\end{array}$ & $\begin{array}{l}\text { T: Net generation efficiency, average annual availability, } \\
\text { construction period, electrical generation potential } \\
\text { Ec: specific investment, cost-benefit index, percentage of } \\
\text { imported inputs } \\
\text { En: water consumption, specific } \mathrm{CO}_{2} \text { emissions, occupied } \\
\text { area, percentage effective land use, specific emissions of } \\
\text { non- } \mathrm{CO}_{2} \text { gas emissions } \\
\text { S: number of direct jobs created, average job income level, } \\
\text { job seasonality }\end{array}$ & $\mathrm{N}$ & $\mathrm{N}$ & $\begin{array}{l}15 \\
M\end{array}$ & $\begin{array}{l}\text { Data envelope } \\
\text { analysis (DEA) }\end{array}$ \\
\hline $\begin{array}{c}\text { Terrados et al. } \\
\text { [39] }\end{array}$ & $\begin{array}{l}\text { Contribute to renewable } \\
\text { energies development at } \\
\text { regional level }\end{array}$ & $\begin{array}{l}\text { T: total primary energy saved, maturity of technology, } \\
\text { technical know-how of local actors, continuity and } \\
\text { predictability of resources } \\
\text { En: } \mathrm{CO}_{2} \text {, other emissions }\left(\mathrm{SO}_{2}, \mathrm{NO}_{\mathrm{x}}\right) \text {, other impacts (noise, } \\
\text { visual impact, landscape) } \\
\mathrm{S} \text { - Ec: job creation, financial requirements, compatibility } \\
\text { with local, regional and national policies }\end{array}$ & $\mathrm{N}$ & $\mathrm{N}$ & $\begin{array}{l}11 \\
\text { QN }\end{array}$ & PROMETHEE \\
\hline $\begin{array}{l}\text { Chatzimou- } \\
\text { ratidis et al. } \\
\quad[40]\end{array}$ & $\begin{array}{l}\text { Evaluation of types of power } \\
\text { plant }\end{array}$ & $\begin{array}{l}\text { T - sustainability: efficiency coefficient, availability, } \\
\text { capacity, reserves/ production (R/P) ratio } \\
\text { Ec: capital cost, fixed O\&M cost, variable O\&M cost, fuel } \\
\text { cost, external cost }\end{array}$ & $\mathrm{N}$ & $\mathrm{N}$ & $\begin{array}{c}8 \\
\text { QN }\end{array}$ & AHP \\
\hline $\begin{array}{l}\text { Van Alphen et } \\
\quad \text { al. [41] }\end{array}$ & $\begin{array}{l}\text { Quantification and } \\
\text { evaluation of the potentials } \\
\text { of available PV and wind }\end{array}$ & $\begin{array}{l}\text { T: excess electricity, renewable energy faction } \\
\text { Ec: capital cost, annual cost, NPV, Levelized cost of energy } \\
\text { En: emission reduction } \\
\text { S: fossil fuel savings }\end{array}$ & $\mathrm{N}$ & $\mathrm{N}$ & $\begin{array}{c}8 \\
\text { QN }\end{array}$ & $\begin{array}{l}\text { Weighted sum } \\
\text { (DEFINITE } \\
\text { software) }\end{array}$ \\
\hline
\end{tabular}




\begin{tabular}{|c|c|c|c|c|c|c|}
\hline \multicolumn{7}{|c|}{$\begin{array}{l}\text { TABLE II continued } \\
\text { Category: Policy selection }\end{array}$} \\
\hline $\begin{array}{c}\text { Kahraman et al. } \\
\text { [42] }\end{array}$ & $\begin{array}{l}\text { Select the best energy policy } \\
\text { alternative }\end{array}$ & $\begin{array}{l}\text { T: feasibility, risk, reliability, duration of preparation phase, } \\
\text { duration of implementation phase, continuity and } \\
\text { predictability of performance, local technical know how } \\
\text { En: pollutant emissions, land requirements, need of waste } \\
\text { disposal } \\
\text { Ec: implementation cost, availability of funds, economic } \\
\text { value (IRR, cost/benefit) } \\
\text { S: compatibility with national energy policy objectives, } \\
\text { political acceptance, social acceptance, labor impact }\end{array}$ & $\mathrm{N}$ & $\mathrm{N}$ & $\begin{array}{l}16 \\
\mathrm{QL}\end{array}$ & Fuzzy AHP \\
\hline Yi et al. [43] & $\begin{array}{l}\text { Solutions to overcome North } \\
\text { Koreas chronic energy } \\
\text { shortage }\end{array}$ & $\begin{array}{l}\text { Ec: facility construction cost, facility maintenance cost, } \\
\text { related infrastructure construction cost } \\
\text { Benefit [S]: availability of energy source within North } \\
\text { Korea, area development of North Korea, improvement of } \\
\text { inter-Korean relations, development of related industry in } \\
\text { South Korea } \\
\text { Risk [T]: technology transfer problem, appropriateness to } \\
\text { North Korea, technological availability and readiness in } \\
\text { South Korea }\end{array}$ & $\mathrm{N}$ & $\mathrm{N}$ & $\begin{array}{l}10 \\
\mathrm{QL}\end{array}$ & AHP \\
\hline $\begin{array}{l}\text { Blechinger, et } \\
\text { al. }[44]\end{array}$ & $\begin{array}{l}\text { Ascertain preferences for } \\
\text { policy measures and } \\
\text { instruments }\end{array}$ & $\begin{array}{l}\text { En: direct contribution to GHG mitigation, indirect } \\
\text { environmental effect } \\
\text { Political acceptability: cost efficiency, dynamic cost, } \\
\text { competiveness, equity, flexibility, stringency for } \\
\text { non-compliance } \\
\text { Feasibility of implementation: implementation network } \\
\text { capacity, administrative feasibility, financial feasibility }\end{array}$ & $\mathrm{N}$ & $\mathrm{N}$ & $\begin{array}{l}10 \\
\mathrm{QL}\end{array}$ & $\begin{array}{l}\text { AHP and Simple } \\
\text { Multi-Attribute } \\
\text { Rating Technique } \\
\text { (SMART) }\end{array}$ \\
\hline \multicolumn{7}{|c|}{ Category: Scenario evaluation } \\
\hline $\begin{array}{c}\text { Diakoulaki et } \\
\text { al. [45] }\end{array}$ & $\begin{array}{l}\text { Examine scenarios for the } \\
\text { expansion of electricity } \\
\text { system }\end{array}$ & $\begin{array}{l}\text { T: guaranteed energy, available power during peak load, } \\
\text { security of supply } \\
\text { Ec: investment cost, production cost } \\
\text { En: } \mathrm{CO}_{2} \text { increase, } \mathrm{SO}_{2}, \mathrm{NO}_{\mathrm{x}}\end{array}$ & $\mathrm{N}$ & $\mathrm{N}$ & $\begin{array}{c}8 \\
\mathrm{M}\end{array}$ & PROMETHEE \\
\hline $\begin{array}{l}\text { Georgopoulou } \\
\text { et al. }[46]\end{array}$ & $\begin{array}{l}\text { Choose among alternative } \\
\text { energy policies at regional } \\
\text { level }\end{array}$ & $\begin{array}{l}\text { T: safety in covering peak load demand, operationality, } \\
\text { stability of the network } \\
\text { Ec: investment cost, O\&M cost } \\
\text { Political: cohesion to local economic activities, regional } \\
\text { employment } \\
\text { En: air quality, noise, visual amenity, depletion of finite } \\
\text { energy sources, risk of climate change, ecosystem's } \\
\text { protection, land use, implementation of EU and national } \\
\text { environmental policy }\end{array}$ & $\mathrm{N}$ & $\mathrm{N}$ & $\begin{array}{l}15 \\
\mathrm{M}\end{array}$ & ELECTRE III \\
\hline \multicolumn{7}{|c|}{ Category: Technology Selection } \\
\hline $\begin{array}{c}\text { Afgan et al. } \\
\text { [12] }\end{array}$ & $\begin{array}{l}\text { Define energy } \\
\text { indicators used in the } \\
\text { assessment of energy } \\
\text { systems which meet } \\
\text { sustainability criterion }\end{array}$ & $\begin{array}{l}\text { Resource: Efficiency } \\
\text { En: Installation cost, electricity cost } \\
\text { Ec: } \mathrm{CO}_{2} \text { emissions } \\
\text { S: Area }\end{array}$ & $\mathrm{Y}$ & $\mathrm{N}$ & $\begin{array}{c}5 \\
\text { QN }\end{array}$ & $\begin{array}{l}\text { Weighted } \\
\text { arithmetic mean }\end{array}$ \\
\hline $\begin{array}{c}\text { Baysal et al. } \\
\quad[13]\end{array}$ & $\begin{array}{l}\text { Selection of renewable } \\
\text { energy power plant } \\
\text { technologies }\end{array}$ & $\begin{array}{l}\text { T: construction period, technical lifetime, capacity factor, } \\
\text { maximum availability } \\
\text { Ec: investment cost, fixed and variable O\&M cost, progress } \\
\text { ratio }\end{array}$ & $\mathrm{Y}$ & $\mathrm{N}$ & $\begin{array}{c}7 \\
\mathrm{QL}\end{array}$ & $\begin{array}{l}\text { Fizzy data } \\
\text { envelopment } \\
\text { analysis (FDEA) }\end{array}$ \\
\hline $\begin{array}{l}\text { Beccali et al. } \\
\text { [21] }\end{array}$ & $\begin{array}{l}\text { Diffusion of renewable } \\
\text { energy technologies at } \\
\text { regional scale }\end{array}$ & $\begin{array}{l}\text { T: Targets of primary energy saving in regional scale, } \\
\text { Technical maturity \& reliability, Consistence of installation } \\
\text { and maintenance requirements with local technical } \\
\text { know-how, Continuity and predictability of performances, } \\
\text { Cost of saved primary energy } \\
\text { Energy and En: Sustainability according to greenhouse } \\
\text { pollutant emissions, Sustainability according to other } \\
\text { pollutant emissions, Land requirement, Sustainability } \\
\text { according to other environmental impacts } \\
\mathbf{S}-\text { Ec: Labor impact, Market maturity, Compatibility with } \\
\text { political, legislative and administrative situation }\end{array}$ & $\mathrm{N}$ & $\mathrm{N}$ & $\begin{array}{l}13 \\
\mathrm{M}\end{array}$ & ELECTRE \\
\hline $\begin{array}{c}\text { Tsoutsos et al. } \\
\text { [47] }\end{array}$ & $\begin{array}{l}\text { Multi-criteria methodology } \\
\text { for sustainable energy } \\
\text { planning on the island of } \\
\text { Crete }\end{array}$ & $\begin{array}{l}\text { T-Ec: Investment, O\&M cost, conventional fuel savings, } \\
\text { maturity of technology, safety of supply } \\
\mathbf{S}-\mathbf{E n}: \mathrm{CO}_{2} \text { emissions avoided, contribution to local } \\
\text { development and welfare, social acceptance and viability of } \\
\text { the remaining environmental effects }\end{array}$ & $\mathrm{N}$ & $\mathrm{N}$ & $\begin{array}{c}8 \\
\mathrm{M}\end{array}$ & PROMETHEE \\
\hline $\begin{array}{l}\text { Burton et al. } \\
\quad[48]\end{array}$ & $\begin{array}{l}\text { Comparison of small scale } \\
\text { schemes with large-scale } \\
\text { alternatives }\end{array}$ & $\begin{array}{l}\text { T: generation capacity, lifespan } \\
\text { Ec: capital cost, O\&M cost } \\
\text { En: } \mathrm{CO}_{2} \text {, impact upon natural environment } \\
\text { S: noise, social effects }\end{array}$ & $\mathrm{N}$ & $\mathrm{N}$ & $\begin{array}{c}8 \\
\mathrm{M}\end{array}$ & MACBETH \\
\hline$\underset{\text { C49] }}{\text { Cavallaro et al. }}$ & $\begin{array}{l}\text { Feasibility assessment to } \\
\text { install wind turbines }\end{array}$ & $\begin{array}{l}\text { T }- \text { Ec: investment cost, O\&M cost, energy production } \\
\text { capacity, savings of finite energy sources, maturity of } \\
\text { technology, realization time } \\
\text { En: } \mathrm{CO}_{2} \text { emissions avoided, visual impact, acoustic noise, } \\
\text { impact on eco-system, social acceptability }\end{array}$ & $\mathrm{N}$ & $\mathrm{N}$ & $\begin{array}{l}11 \\
\mathrm{M}\end{array}$ & NAIADE \\
\hline
\end{tabular}




\begin{tabular}{|c|c|c|c|c|c|c|}
\hline \multicolumn{7}{|c|}{ TABLE II continued } \\
\hline $\begin{array}{c}\text { Afgan et al. } \\
\text { [50] }\end{array}$ & $\begin{array}{l}\text { Assessment of hydrogen } \\
\text { energy options in } \\
\text { comparison with renewables }\end{array}$ & $\begin{array}{l}\text { Performance: Efficiency, electricity cost, capital cost, life } \\
\text { time } \\
\text { Market: European market, world market } \\
\text { En: } \mathrm{CO}_{2}, \mathrm{NO}_{x}, \text { Kyoto indicator } \\
\text { S: area, new jobs }\end{array}$ & $\mathrm{N}$ & $\mathrm{Y}$ & $\begin{array}{l}11 \\
\mathrm{QN}\end{array}$ & $\begin{array}{l}\text { Weighted } \\
\text { arithmetic mean }\end{array}$ \\
\hline $\begin{array}{l}\text { Afgan } e t a l . \\
\text { [51] }\end{array}$ & $\begin{array}{l}\text { Evaluation of hybrid energy } \\
\text { systems }\end{array}$ & $\begin{array}{l}\text { Ec: Efficiency, electricity cost, investment cost } \\
\text { En: } \mathrm{CO}_{2} \text { emissions } \\
\text { S: } \mathrm{NO}_{\mathrm{x}} \text { emissions }\end{array}$ & $\mathrm{N}$ & $\mathrm{Y}$ & $\begin{array}{c}5 \\
\mathrm{QN}\end{array}$ & $\begin{array}{l}\text { Weighted } \\
\text { arithmetic mean of } \\
\text { indicators }\end{array}$ \\
\hline $\begin{array}{l}\text { Mourmouris et } \\
\quad \text { al. [52] }\end{array}$ & $\begin{array}{l}\text { Support energy planning for } \\
\text { promoting the use of RETs }\end{array}$ & $\begin{array}{l}\text { T: efficiency, safety, availability, } \\
\text { En: environmental quality, visual impacts, impacts on flora } \\
\text { and fauna, } \mathrm{CO}_{2}, \mathrm{SO}_{2}, \mathrm{NO}_{\mathrm{x}} \text { emissions } \\
\text { Ec: economic benefits for the region, creation of } \\
\text { development } \\
\text { S: employment in the energy sector, land used, social } \\
\text { acceptability }\end{array}$ & $\mathrm{N}$ & $\mathrm{N}$ & $\begin{array}{l}14 \\
\mathrm{M}\end{array}$ & REGIME \\
\hline $\begin{array}{l}\text { Topcu et al. } \\
\quad[53]\end{array}$ & $\begin{array}{l}\text { Selection of suitable } \\
\text { electricity generation } \\
\text { alternatives }\end{array}$ & $\begin{array}{l}\text { P: sustainability of the energy resource, suitability of } \\
\text { potential site } \\
\text { En: Externality cost } \\
\text { Ec: Levelized cost } \\
\text { Political and uncontrollable: stability }\end{array}$ & $\mathrm{N}$ & $\mathrm{N}$ & $\begin{array}{c}5 \\
M\end{array}$ & PROMETHEE \\
\hline $\begin{array}{l}\text { Kaldellis et al. } \\
\text { [54] }\end{array}$ & $\begin{array}{l}\text { Provide decision makers } \\
\text { with tool to evaluate } \\
\text { technologies to support } \\
\text { power generation }\end{array}$ & $\begin{array}{l}\text { T: system efficiency, capacity factor, fuel availability, } \\
\text { existing experience } \\
\text { Ec: } 14 \text { criteria, i.e. high paid cost/tn of } \mathrm{CO}_{2} \text {, fuel cost, } \\
\text { construction cost/long payback period, etc. } \\
\text { En: } 14 \text { criteria, i.e. high gaseous and particulate emissions, } \\
\text { hot waste water disposal, microclimate change, etc. } \\
\text { S: } 9 \text { criteria, i.e. noise, accidents, health hazards, people } \\
\text { relocation etc. }\end{array}$ & $\mathrm{N}$ & $\mathrm{N}$ & $\begin{array}{l}41 \\
\mathrm{QL}\end{array}$ & $\begin{array}{l}\text { Delphi method } \\
\text { (qualitative } \\
\text { evaluating } \\
\text { approach) }\end{array}$ \\
\hline Kaya et al. [55] & $\begin{array}{l}\text { 1) determining best } \\
\text { renewable energy alternative } \\
\text { 2) selecting site location }\end{array}$ & $\begin{array}{l}\text { T: technical efficiency, exergy efficiency } \\
\text { Ec: Investment cost, } \mathrm{O} \& \mathrm{M} \\
\text { En: } \mathrm{NO}_{\mathrm{x}} \text { emissions, } \mathrm{CO}_{2} \text { emissions, land use } \\
\text { S: social acceptability, job creation }\end{array}$ & $\mathrm{N}$ & $\mathrm{N}$ & $\begin{array}{c}9 \\
\mathrm{QL}\end{array}$ & $\begin{array}{l}\text { integrated fuzzy } \\
\text { VIKOR-AHP } \\
\text { algorithm }\end{array}$ \\
\hline $\begin{array}{c}\text { Evans et al. } \\
\quad[56]\end{array}$ & $\begin{array}{l}\text { Assessment of RETs using } \\
\text { sustainability indicators }\end{array}$ & $\begin{array}{l}\text { T: Availability and limitations, efficiency } \\
\text { Ec: Price } \\
\text { En: } \mathrm{CO}_{2} \text { emissions, land use, water consumption } \\
\text { S: social impacts }\end{array}$ & $\mathrm{N}$ & $\mathrm{N}$ & $\begin{array}{c}7 \\
\mathrm{QL}\end{array}$ & Equal weights \\
\hline Onat et al. [57] & $\begin{array}{l}\text { Assessment of electricity } \\
\text { generating technologies }\end{array}$ & $\begin{array}{l}\text { T: Availability, efficiency } \\
\text { Ec: Unit energy cost } \\
\text { En: } \mathrm{CO}_{2} \text { emissions, land use, fresh water consumption } \\
\text { S: external costs, external benefits }\end{array}$ & $\mathrm{N}$ & $\mathrm{N}$ & $\begin{array}{l}8 \\
\mathrm{M}\end{array}$ & $\begin{array}{l}\text { Ranking of criteria } \\
\text { and equal weights }\end{array}$ \\
\hline $\begin{array}{l}\text { Varun et al. } \\
\quad[58]\end{array}$ & $\begin{array}{l}\text { Technology selection for } \\
\text { sustainable development }\end{array}$ & $\begin{array}{l}\text { T: power rating, life } \\
\text { Ec: energy pay-back time, cost of electricity generation } \\
\text { En: GHG emissions }\end{array}$ & $\mathrm{N}$ & $\mathrm{N}$ & $\begin{array}{c}5 \\
\mathrm{QN}\end{array}$ & $\begin{array}{l}\text { Figure of merit } \\
\text { based on equal } \\
\text { weighting }\end{array}$ \\
\hline Cavallaro[59] & $\begin{array}{l}\text { Preliminary } \\
\text { assessment of CSP } \\
\text { technologies }\end{array}$ & $\begin{array}{l}\text { T: maturity of technology, temperature, solar capacity factor } \\
\text { Ec: investment cost, O\&M cost, LEC } \\
\text { En: environmental impact }\end{array}$ & $\mathrm{N}$ & $\mathrm{N}$ & $\begin{array}{l}7 \\
M\end{array}$ & $\begin{array}{l}\text { PROMETHEE, } \\
\text { GAIA }\end{array}$ \\
\hline $\begin{array}{c}\text { Buchholz et al. } \\
{[60]}\end{array}$ & $\begin{array}{l}\text { Assess sustainability of } \\
\text { bioenergy systems with } \\
\text { focus on multi-stakeholder } \\
\text { inclusion }\end{array}$ & $\begin{array}{l}\text { Ecological: reduced competition for fertile land } \\
\text { Ec: increased local commerce, high cost efficiency, high } \\
\text { supply security } \\
\text { S: low training needs, high employment rate, diversity and } \\
\text { certainty in ownership and business schemes, low planning } \\
\text { and monitoring needs }\end{array}$ & $\mathrm{N}$ & $\mathrm{N}$ & $\begin{array}{c}8 \\
\mathrm{QL}\end{array}$ & $\begin{array}{l}\text { AHP, MAUT, } \\
\text { PROMETHEE and } \\
\text { NAIADE }\end{array}$ \\
\hline $\begin{array}{l}\text { Pilavachi et al. } \\
\text { [61] }\end{array}$ & $\begin{array}{l}\text { Evaluate electrical energy } \\
\text { generation options }\end{array}$ & $\begin{array}{l}\text { T: efficiency, service of life } \\
\text { En: } \mathrm{CO}_{2} \text { emissions, } \mathrm{NO}_{\mathrm{x}} \text { emissions } \\
\text { Ec: capital cost, } \mathrm{O} \& \mathrm{M} \text { costs, electricity cost }\end{array}$ & $\mathrm{N}$ & $\mathrm{N}$ & $\begin{array}{c}7 \\
\text { QN }\end{array}$ & AHP \\
\hline Erol et al. [62] & $\begin{array}{l}\text { Facilitate energy resource } \\
\text { planning activities }\end{array}$ & $\begin{array}{l}\text { T: possibility of acquiring original technology, superiority } \\
\text { of technology, completeness of technology } \\
\text { Ec: reliability of technology and operation, ease of access to } \\
\text { the source, additional investment, source durability, } \\
\text { supplementary usage of resources } \\
\text { En: effect of the technology to the environment, carbon } \\
\text { footprint, requirement of resources } \\
\text { Public: acceptability by local resident }\end{array}$ & $\mathrm{N}$ & $\mathrm{N}$ & $\begin{array}{l}12 \\
\mathrm{QL}\end{array}$ & AHP \\
\hline$\underset{[63]}{\text { Nigim et al. }}$ & $\begin{array}{l}\text { Assist communities in } \\
\text { prioritizing their RES } \\
\text { alternatives }\end{array}$ & $\begin{array}{l}\text { Ecological impact, social and economic benefits, } \\
\text { educational potential, Resource availability, technical } \\
\text { feasibility, financial feasibility }\end{array}$ & $\mathrm{N}$ & $\mathrm{N}$ & $\begin{array}{c}6 \\
\mathrm{QL}\end{array}$ & $\begin{array}{l}\text { AHP and SIMUS } \\
\text { tool }\end{array}$ \\
\hline $\begin{array}{l}\text { Streimikiene } e t \\
\text { al. [64] }\end{array}$ & $\begin{array}{l}\text { Choosing the most } \\
\text { sustainable } \\
\text { electricity generation } \\
\text { technologies }\end{array}$ & $\begin{array}{l}\text { Ec: private costs, average availability (load) factor, security } \\
\text { of supply, costs of grid connection, peal load response } \\
\text { En: GHG emissions, environmental external costs, } \\
\text { radionuclide external cost, human health impact } \\
\text { S: technology-specific job opportunities, food safety risk, } \\
\text { fatal accidents from the past experience, severe accidents } \\
\text { perceived in future }\end{array}$ & $\mathrm{N}$ & $\mathrm{N}$ & $\begin{array}{l}13 \\
M\end{array}$ & $\begin{array}{l}\text { Multimoora and } \\
\text { TOPSIS }\end{array}$ \\
\hline $\begin{array}{c}\text { San Cristobal } \\
{[65]}\end{array}$ & $\begin{array}{l}\text { Selection of a Renewable } \\
\text { Energy investment project }\end{array}$ & $\begin{array}{l}\text { T: power, operating hours, implementation period, useful } \\
\text { life } \\
\text { Ec: investment ratio, O\&M costs } \\
\text { En: tons of } \mathrm{CO}_{2} \text { avoided }\end{array}$ & $\mathrm{N}$ & $\mathrm{N}$ & $\begin{array}{c}7 \\
\text { QN }\end{array}$ & $\begin{array}{l}\text { Compromise } \\
\text { ranking method } \\
\text { VIKOR }\end{array}$ \\
\hline
\end{tabular}




\begin{tabular}{|c|c|c|c|c|c|c|}
\hline \multicolumn{7}{|c|}{ TABLE II continued } \\
\hline \multicolumn{7}{|c|}{ Category: Site selection } \\
\hline$\underset{[24]}{\text { Al-Yahyai } \text { et al. }}$ & $\begin{array}{l}\text { Derive wind farm land } \\
\text { suitability index and } \\
\text { classification }\end{array}$ & $\begin{array}{l}\text { T: wind power density, energy demand matching, } \\
\text { percentage of sustainable wind, turbulence intensity, sand } \\
\text { dunes } \\
\text { Ec: distance to road, terrain slope } \\
\text { En: historical locations, wildlife and natural reserves } \\
\text { S: urban area }\end{array}$ & $\mathrm{N}$ & $\mathrm{N}$ & $\begin{array}{l}10 \\
\mathrm{M}\end{array}$ & $\begin{array}{l}\text { AHP with Ordered } \\
\text { Weigh Averaging }\end{array}$ \\
\hline $\begin{array}{c}\text { Charabi et al. } \\
\text { [25] }\end{array}$ & $\begin{array}{l}\text { Assess the land suitability } \\
\text { for large PV farms } \\
\text { implementation }\end{array}$ & $\begin{array}{l}\text { T: solar radiation, land accessibility, land use } \\
\text { Ec: grid proximity, land slope, load poles } \\
\text { En: sensitive areas, hydrographic line, sand/dusk risk }\end{array}$ & $\mathrm{N}$ & $\mathrm{N}$ & $\begin{array}{c}9 \\
M\end{array}$ & $\begin{array}{l}\text { Fuzzy Logic } \\
\text { Ordered Weighted } \\
\text { Averaging } \\
\text { (FLOWA) }\end{array}$ \\
\hline $\begin{array}{l}\text { Defne et al. } \\
\quad \text { [26] }\end{array}$ & $\begin{array}{l}\text { Assist in selecting most } \\
\text { suitable locations for tidal } \\
\text { stream projects }\end{array}$ & $\begin{array}{l}\text { Physical: power density } \\
\text { En: environmental score } \\
\text { S-Ec: accessibility }\end{array}$ & $\mathrm{Y}$ & $\mathrm{N}$ & $\begin{array}{c}3 \\
M\end{array}$ & $\begin{array}{l}\text { GIS and equal } \\
\text { weighting }\end{array}$ \\
\hline $\begin{array}{c}\text { Haurant et al. } \\
{[66]}\end{array}$ & $\begin{array}{l}\text { Selection of photovoltaic } \\
\text { plant projects }\end{array}$ & $\begin{array}{l}\text { T: net production } \\
\text { Geoeconomic: rent area unoccupied by the installation } \\
\text { Ecological: study of the potential ecological degradation in } \\
\text { the files } \\
\text { En: relevance of visual impact presentation in the files, } \\
\text { observer-plant minimum distance } \\
\text { Territorial use: use conflicts risks } \\
\text { Ec: economic activity and inhabitants' financial benefits } \\
\text { related to RES facilities, financial incomes at the communal } \\
\text { level }\end{array}$ & $\mathrm{N}$ & $\mathrm{N}$ & $\begin{array}{c}8 \\
\mathrm{M}\end{array}$ & ELECTRE \\
\hline $\begin{array}{l}\text { Zhang et al. } \\
\text { [67] }\end{array}$ & $\begin{array}{l}\text { Selecting a sustainable } \\
\text { energy plan for Nanjing city }\end{array}$ & $\begin{array}{l}\text { T: efficiency, safety, reliability } \\
\text { Ec: investment cost, O\&M cost } \\
\text { En: GHG emissions reduction, land use } \\
\text { S: job creation, social benefit }\end{array}$ & $\mathrm{N}$ & $\mathrm{N}$ & $\begin{array}{c}8 \\
\mathrm{QL}\end{array}$ & $\begin{array}{l}\text { Fuzzy integral } \\
\text { method }\end{array}$ \\
\hline \multicolumn{7}{|c|}{ Category: Storage technology selection } \\
\hline Barin et al. [15] & $\begin{array}{l}\text { Evaluate operation of } \\
\text { storage energy } \\
\text { systems }\end{array}$ & $\begin{array}{l}\text { T: efficiency, load management, technical maturity, } \\
\text { lifecycle, power quality } \\
\text { Ec: costs }\end{array}$ & $\mathrm{N}$ & $\mathrm{Y}$ & $\begin{array}{c}6 \\
M\end{array}$ & $\begin{array}{l}\text { AHP and fuzzy } \\
\text { sets }\end{array}$ \\
\hline Raza et al. [16] & $\begin{array}{l}\text { Compare different energy } \\
\text { storage systems for their } \\
\text { sustainability }\end{array}$ & $\begin{array}{l}\text { T: fast load response capability, reliability, system life, } \\
\text { efficiency, capacity or efficiency variation, risk factor, } \\
\text { modularity production, energy density ratio } \\
\text { En: cost } \\
\text { Ec: environmental impact }\end{array}$ & $\mathrm{N}$ & $\mathrm{Y}$ & $\begin{array}{l}10 \\
\mathrm{M}\end{array}$ & $\begin{array}{l}\text { Sustainable Index } \\
\text { approach using } \\
\text { weighted sum }\end{array}$ \\
\hline \multicolumn{7}{|c|}{$\begin{array}{l}\text { Abbreviations: } \mathrm{N}=\mathrm{No} ; \mathrm{Y}=\text { Yes; } \mathrm{T}=\text { Technical; En = Environmental; Ec = Economic; } \mathrm{S}=\text { Social; } \mathrm{I}=\text { Institutional; } \mathrm{P}=\mathrm{Physical} ; \mathrm{T}-\mathrm{Ec}= \\
\text { Techno-economic; } \mathrm{S}-\mathrm{Ec}=\mathrm{Socio}-\mathrm{economic} ; \mathrm{S}-\mathrm{En}=\text { Socio-environmental } \\
\mathrm{NPV}=\text { Net Present Value; } \mathrm{O} \& \mathrm{M}=\text { Operation and Maintenance; } \mathrm{PP}=\text { Payback period; } \mathrm{CSP}=\text { Concentrated solar power; } \mathrm{LEC}=\text { Levelized electricity cost } \\
\text { Data type can be quantitative }(\mathrm{QN}) \text {, qualitative }(\mathrm{QL}) \text { or mixed }(\mathrm{M}) \\
\text { *The different MCDM methods are not further explained within this paper. Precise information about each method can be found under the respective } \\
\text { references. }\end{array}$} \\
\hline
\end{tabular}

The review of MCDM for renewable energy and storage systems highlights:

- Most research is undertaken for technology selections and power system optimization;

- Offshore and storage technologies lack consideration;

- Combinations of offshore RET and storage technologies could not be found

- Criteria vary, but the most common are: technical, environmental, economic and social

- The chosen indicators also diverge strongly (the most common by criteria are; technical: efficiency, availability and lifetime; economic: investment cost and O\&M cost; environmental: $\mathrm{CO}_{2}$ emissions and land use; social: job creation, acceptability);

- The majority of researches uses mixed data sets with an average set of 8-10 indicators;

- AHP is the most frequently applied method in the analyzed researches

When it comes to the applicability of reviewed studies (Table III), immediately the high focus on PV $(>75 \%)$ and wind $(>70 \%)$ becomes evident. Moreover, biomass, hydro and non-renewables are assessed in around $50 \%$ of the researches. Hydrogen and offshore technologies only play a marginal role. In terms of system size various studies focus on island energy systems, but with a limited number of technologies considered. Moreover, it is essential to highlight that none of the studies focused on all technologies. Indeed, out of 47 studies reviewed only twice offshore technologies were considered.

\section{DisCusSiOn ON Future ENERGy PlanNing CONSIDERATIONS FOR ISLANDS}

Owing to its particular, often natural, conditions, isolated islands require a more acquainted usage of renewable resources. Case specific energy planning and management is highly advisable, because each island can be considered as an individual and differing energy system.

The following conductions will reflect upon such a system and point out important aspects to be considered for island energy planning. Regarding the system size, small not grid-connected islands ranging from a few thousand up to a million inhabitants shall be accounted for.

\section{A. Future Development and Energy Time Shift}

While an islands energy demand often fluctuates by a few folds within a year, long-term planning considers time horizons of 25 years and more. In this time frame noticeable changes can occur within an island energy system; either increasing or decreasing. Consequently, forecasts are vital to define the compulsory future energy requirements. Such investigation should also consider by how much energy efficiency can decrease consumption and by how much 
demand changes due to an increase or decrease of habitants.

Besides, it should be a major interest to assess the possibilities of energy management. On the one hand side, typical demand side measures are peak clipping, valley lifting, load shifting, strategic conversation, strategic load growth or a flexible load shape. Indeed, high flexibility in the load shape is essential to cope with variable renewables. On the other side, supply side management is desirable. Thereby, the focus is placed on efficiency along the whole value chain, ranging from generation to distribution and the final customer. Smart technology and smart grids are the connection between demand and supply and therefore possess a major role within the planning and secure energy supply.

TABLE III: EVALUATION OF SySTEM Size AND TeChNOLOGY

\begin{tabular}{|c|c|c|c|c|c|c|c|c|c|c|c|}
\hline \multirow[t]{2}{*}{ Ref. } & \multirow[t]{2}{*}{$\begin{array}{l}\text { Applied system size and location } \\
\text { (Comment) }\end{array}$} & \multicolumn{6}{|c|}{ Onshore } & \multicolumn{3}{|c|}{ Offshore } & \multirow[t]{2}{*}{$\begin{array}{l}\text { Fossil } \\
\text { fuels }\end{array}$} \\
\hline & & $\mathrm{S}$ & W & HY & B & G & HG & $\mathrm{W}$ & WA & $\mathrm{T}$ & \\
\hline [12] & Any - conceptual method & $\checkmark$ & $\checkmark$ & $\checkmark$ & $\checkmark$ & $\checkmark$ & $\checkmark$ & & & & $\checkmark$ \\
\hline [13] & Technology specific selection & $\checkmark$ & $\checkmark$ & $\checkmark$ & & $\checkmark$ & & $\checkmark$ & & & \\
\hline [21] & Regional, island of Sardinia, Italy & $\checkmark$ & $\checkmark$ & $\checkmark$ & $\checkmark$ & & & & & & $\checkmark$ \\
\hline [24] & Local, regional, national, Oman & & $\checkmark$ & & & & & & & & \\
\hline$[25]$ & Local, regional, national, Oman & $\checkmark$ & & & & & & & & & \\
\hline [26] & Local, regional, coast of Georgia & & & & & & & & & $\checkmark$ & \\
\hline$[27]$ & $\begin{array}{l}\text { Off-grid, rural, Pangan-an Island, } \\
\text { Philippines }\end{array}$ & $\checkmark$ & & & & & & & & & \\
\hline [28] & $\begin{array}{l}\text { Remote areas, rural, } \\
\text { non-interconnected zones Columbia }\end{array}$ & $\checkmark$ & $\checkmark$ & $\checkmark$ & $\checkmark$ & & & & & & $\checkmark$ \\
\hline [29] & $\begin{array}{l}\text { Rural livelihoods, communities, San } \\
\text { Jose, Colombia }\end{array}$ & $\checkmark$ & & $\checkmark$ & $\checkmark$ & & & & & & $\checkmark$ \\
\hline [30] & National, Korea & & & & & & & & & & \\
\hline$[31]$ & $\begin{array}{l}\text { Local community level up to national } \\
\text { level }\end{array}$ & $\checkmark$ & $\checkmark$ & $\checkmark$ & $\checkmark$ & $\checkmark$ & & & & & $\checkmark$ \\
\hline$[32]$ & $\begin{array}{l}\text { Rural livelihoods, communities, } \\
\text { Jambaló, Colombia }\end{array}$ & $\checkmark$ & & $\checkmark$ & $\checkmark$ & & & & & & $\checkmark$ \\
\hline$[33]$ & $\begin{array}{l}\text { Any - Power system optimization } \\
\text { model }\end{array}$ & $\checkmark$ & $\checkmark$ & $\checkmark$ & $\checkmark$ & $\checkmark$ & & & & & $\checkmark$ \\
\hline [34] & Large, national, Portugal & & $\checkmark$ & $\checkmark$ & & & & & & & $\checkmark$ \\
\hline [35] & Regional to national - Bosnia & $\checkmark$ & $\checkmark$ & $\checkmark$ & $\checkmark$ & & & & & & $\checkmark$ \\
\hline [36] & Local, regional, power stations & & $\checkmark$ & $\checkmark$ & $\checkmark$ & & & & & & $\checkmark$ \\
\hline$[37]$ & $\begin{array}{l}\text { Remote region, isolated Greek islands } \\
\text { Karpathos and Kassos }\end{array}$ & $\checkmark$ & $\checkmark$ & & $\checkmark$ & & & & & & \\
\hline [38] & Any - conceptual method & & $\checkmark$ & $\checkmark$ & $\checkmark$ & & & & & & \\
\hline [39] & Local, regional, Province Jaen, Spain & $\checkmark$ & $\checkmark$ & $\checkmark$ & $\checkmark$ & & & & & & $\checkmark$ \\
\hline [40] & Local, regional to national & $\checkmark$ & $\checkmark$ & $\checkmark$ & $\checkmark$ & $\checkmark$ & & & & & $\checkmark$ \\
\hline$[41]$ & $\begin{array}{l}\text { Local, regional, city, islands, Male, } \\
\text { Maldives }\end{array}$ & $\checkmark$ & $\checkmark$ & & & & & & & & \\
\hline [42] & National, Turkey & $\checkmark$ & $\checkmark$ & $\checkmark$ & $\checkmark$ & $\checkmark$ & & & & & \\
\hline [43] & National, North Korea & $\checkmark$ & $\checkmark$ & $\checkmark$ & $\checkmark$ & $\checkmark$ & & & & & $\checkmark$ \\
\hline$[44]$ & $\begin{array}{l}\text { Regional, national policies, Trinidad } \\
\text { and Tobago }\end{array}$ & & & & & & & & & & \\
\hline [45] & National, Greece & & $\checkmark$ & $\checkmark$ & & & & & & & $\checkmark$ \\
\hline [46] & Local, regional level, Crete & $\checkmark$ & $\checkmark$ & & $\checkmark$ & & & & & & $\checkmark$ \\
\hline [47] & Regional, Crete & $\checkmark$ & $\checkmark$ & & $\checkmark$ & & & & & & \\
\hline$[48]$ & $\begin{array}{l}\text { Local, small scale, Borough of } \\
\text { Kirklees in Yorkshire, UK }\end{array}$ & $\checkmark$ & $\checkmark$ & $\checkmark$ & $\checkmark$ & & & & & & $\checkmark$ \\
\hline [49] & $\begin{array}{l}\text { Local to regional, island of Salina, } \\
\text { Italy }\end{array}$ & & $\checkmark$ & & & & & & & & \\
\hline$[50]$ & Any - conceptual method & $\checkmark$ & $\checkmark$ & & & & $\checkmark$ & & & & $\checkmark$ \\
\hline [51] & Any - conceptual method & $\checkmark$ & $\checkmark$ & & $\checkmark$ & & $\checkmark$ & & & & $\checkmark$ \\
\hline [52] & Regional, Thassos, Greece & $\checkmark$ & $\checkmark$ & $\checkmark$ & $\checkmark$ & $\checkmark$ & & & & & \\
\hline [53] & Regional to national, Turkey & $\checkmark$ & $\checkmark$ & $\checkmark$ & $\checkmark$ & & & & & & $\checkmark$ \\
\hline$[54]$ & $\begin{array}{l}\text { Regional and National, Crete and } \\
\text { Greece }\end{array}$ & $\checkmark$ & $\checkmark$ & & & & & & & & $\checkmark$ \\
\hline [55] & Local, city, Istanbul & $\checkmark$ & $\checkmark$ & $\checkmark$ & $\checkmark$ & $\checkmark$ & & & & & \\
\hline [56] & Any - conceptual method & $\checkmark$ & $\checkmark$ & $\checkmark$ & & $\checkmark$ & & & & & \\
\hline [57] & Any - conceptual method & $\checkmark$ & $\checkmark$ & $\checkmark$ & & $\checkmark$ & $\checkmark$ & & & & $\checkmark$ \\
\hline [58] & International/National & $\checkmark$ & $\checkmark$ & $\checkmark$ & & & & & & & \\
\hline [59] & Technology specific selection & $\checkmark$ & & & & & & & & & \\
\hline [60] & Local, regional, national, Uganda & & & & $\checkmark$ & & & & & & $\checkmark$ \\
\hline [61] & Technology specific selection & & & & & & $\checkmark$ & & & & $\checkmark$ \\
\hline$[62]$ & $\begin{array}{l}\text { Local, regional, district of Aydin, } \\
\text { Turkey }\end{array}$ & $\checkmark$ & $\checkmark$ & $\checkmark$ & & $\checkmark$ & & & & & $\checkmark$ \\
\hline$[63]$ & $\begin{array}{l}\text { Local, communities, Waterloo Region, } \\
\text { southern Ontario }\end{array}$ & $\checkmark$ & $\checkmark$ & $\checkmark$ & & $\checkmark$ & & & & & \\
\hline [64] & Technology specific selection & $\checkmark$ & $\checkmark$ & $\checkmark$ & $\checkmark$ & & & & & & $\checkmark$ \\
\hline [65] & Local, regional, national & $\checkmark$ & $\checkmark$ & $\checkmark$ & $\checkmark$ & & & & & & \\
\hline [66] & Local, regional, Corsica Island & $\checkmark$ & & & & & & & & & \\
\hline$[67]$ & Local, city-level, Nanjing, China & $\checkmark$ & & & $\checkmark$ & $\checkmark$ & & & & & \\
\hline \multicolumn{2}{|c|}{ Total (47) } & 36 & 34 & 28 & 26 & 14 & 5 & 1 & $\mathbf{0}$ & 1 & 24 \\
\hline
\end{tabular}




\section{B. Future Development and Energy Vector Shift}

In addition to the changes resulting from energy efficiency and time shifts, the effects of energy vector shifts should be assessed. This is particularly important for small island energy systems. There, large scale heat generation from RETs is impractical. Even with combined heat and power systems large heat sinks might be required during off-peak season. This consideration is vital for decision makers, when planning for a system that is mainly driven by renewable based electricity. Indeed, within $25-50$ years' time it could be a possible scenario to have the bulk of an islands car fleet powered by electricity. Plus, currently fossil fuel driven heating appliances might be electricity driven by then. Certainly, it is not the intension to replace all heat and transport fuel requirements with electricity. Especially, for the building and industry sector different alternatives of heat generation from renewables are worth considering; i.e. solar thermal, biomass, ground source heat pumps, etc.

Due to the vector shift, and despite the energy savings from energy efficiency and management, the overall electricity demand on an island might increase remarkably within the considered time horizon. Hence, the new energy paradigm will create a new load profile.

Thereby, it should be aimed for a flexible load shape, whereas demand in the form of the inhabitant's requirements can match with the energy provided from renewables. Additionally, storage capacity will be required. This can either be storage plants or batteries, but also fossil fuel powered back-up generators. Nonetheless, with fossil fuel back-up the dependency on fossil fuel imports remains. Consequently, the planning should evaluate some type of storage technology or capacity. Due to the small system size the focus is not places on fast responding back-up capacity for system stabilization, but also long-term back-up when there are longer periods without or limited availability of RET based power. In order to reduce the unavailability of a large share of RET, technology diversification is highly encouraged.

\section{Enlarge Number of Technologies within Selection Process}

Choosing the right technology is one of the most important tasks for decision makers when undergoing energy planning for sustainable development. While in the past decades the number of possible REt alternatives was rather small, and technologies could be selected mainly from a commercial point of view, by now, several market commercial concepts are available. Many of them are highly competitive with one another. For that reason more precise targets need to be defined before selecting a technology. Emerging concepts, mainly offshore technologies, should not be neglected in the selection process. Especially in spatially limited islands, but also ones that contain heritage and natural habitats, a focus on offshore rather than onshore RETs is advisable.

Besides, islands do not necessarily seek for the most economic supply alternative, rather than for one that is sustainable. Aspects such as reducing import dependency and thus having energy security are often much higher valued.

Indeed, offshore RETs lack of market competitiveness yet, apart from offshore wind. Hence, the expectations and trends are promising. Offshore resources bear several advantages compared to their onshore counterparts, whereas especially environmental and social aspects could favor offshore technologies.

It is this variety of technologies that confronts decision makers with major challenges; 1) what technology or technology portfolio is most suitable one to cover the energy requirements?, and 2) how to combine RET and storage technologies to have a reliable and secure energy system? For that reason decision support methods are helpful to select appropriate technologies according to the preferences and local requirements of the specific case. Though, such methods should be able to incorporate the preferences of decision makers and allow for a differentiation of available data

\section{New Island Energy Planning Concept}

The discussion has highlighted the need for a holistic approach under the new energy paradigm. Electricity as a dominating energy vector can certainly supply the vast majority of an islands energy requirement with locally abundant resources. Therefore, more precise decision support is required. Diesel generators should only be considered an alternative once the storage system is not able to provide adequate back-up power.

A new concept, based on the conductions of the previous paragraphs is currently under development at the University of Porto. It is intended to develop a method whereby multiple MCDM processes will be undergone to propose a technology portfolio that can cover an islands energy requirements based on a combination of supply alternatives from renewable energy sources. At the present stage the concept is divided in 5 major phases.

\section{1) Define demand and load profiles}

The principal step is to define the current energy demand. Therefore, the peak capacity, annual demand and daily load profiles will be aimed for. As the future demand is subject to a variety of external factors, 9 different scenarios will be built to represent the future demand. Those scenarios are the combinations of three alternatives (conservative increase, progressive increase and very progressive increase) stating the amount of electricity to be covered from renewables along with three time frames (10 years, 20 years and 30 years).

- The conservative approach intends to fulfill the future (additional) electricity demand and whatever results from the phase-out of fossil fuel power plants.

- The progressive approach foresees replacing all fossil fuel power generation.

- Within the very progressive approach all fossil fuel power generation plus all heating and transportation services that can become electric within the specific time horizon shall be covered by renewable energy technologies.

Hence, for each of these 9 scenarios daily load profiles are required, depicting the hourly loads over the year. As precise measurements for everyday are often difficult to access and scarce, the profiles for weekday, Saturday and Sunday over the different months or seasons shall be used. A minimum of 3 load profiles is desired to perform the time series simulation in phase 4.

The following assumptions were reflected when compiling the scenarios. 
- In all scenarios implying the conservative approach there is enough fossil fuel back-up available to integrate RET.

- For all scenarios implying the progressive approach a replacement of all fossil fuel generation by RETs can be done within the proposed time frames.

- The load profiles for all scenarios comprising the conservative or progressive approach are identical and will not change over time.

- All scenarios including the progressive or very progressive approach consider the usage of storage technologies. Suitable amounts of storage will be defined within phase 4 . In order to guarantee $100 \%$ system reliability a minimum amount of fossil fuel back-up power might be required.

- Scenarios comprising the very progressive approach consider different amounts of energy vector shifts to be undertaken over time. Thereby, aproportionally increasing shifting potential will be considered over the time horizons.

- Different load profiles reflecting the service shifts will be applied in the scenarios comprising the very progressive approach.

\section{2) Technology options}

This step intends to evaluate different RETs according to the local resource availability and characteristics. In fact, 36 different onshore and offshore technologies will be assessed. Several emerging concepts, especially wave and tidal, can be added once more precise data is available. While the resource conditions assess the general suitability of the technology, with different technology specific characteristics, the number of possible technologies will be reduced and specified. The evaluation criteria are very technology specific and therefore allow for a more precise technology selection. For instance, within offshore wind there are floating and fixed devices, but due to the water depth around an island only floating devices might be suitable. Plus, within the floating devices various considerations regarding the mooring dynamics, hydrodynamics, aerodynamics and so forth are applied. For onshore technologies the criteria are more related to space limitation, shading or land accessibility.

In a similar manner criteria for each technology category have been defined to detect the most appropriate technology choice within each technology category. This primer multi-criteria approach has been chosen to minimize the amount of technologies to be considered for further evaluation.

\section{3) Sustainability assessment}

The sustainability assessment will be undertaken for all pre-specified technology choices over the three time horizons (10, 20 and 30 years). A family of criteria will be defined to evaluate the value and the consequences of the technologies under technical, economic, environmental and social aspects. Therefore, a MCDM process will be undertaken. Equal weighting is proposed in the first instance. Thereby, all pre-specified technologies will be ranked. Learning curves over the time horizons will be associated to each evaluation indicator; i.e. efficiency improvements, cost reduction, local perception, etc.

In a second approach each of the evaluation indicators can be graded by preferences and priorities; for instance by decision makers or energy agencies. This will give applicants of this concept a higher flexibility in their decision making process.

Once all technologies are ranked accordingly, only the top 5 technologies in each time horizon will be considered for further evaluation. Though, it will be a constraint to not use different technology choices with the same resource. The diversity of technologies is desired as the amount of storage can be reduced and the system becomes more reliable.

\section{4) Determination of energy mix}

At this stage the technologies and their natural resource availability are identified. The latter can be obtained from local weather stations. Additionally, the primarily collected load profiles for each scenario are available. Based on a time-series approach that considers the availability of each resource for each hour of the year along with the demand profile that defines the load for each hour of the year it is intended to define the energy mix that minimizes the maximum amount of RET and storage to be required. As a result, the energy mix for each of the 9 scenarios could consider up to 5 different technologies. At the current stage only one storage technology will be considered to cover the required back-up power. Though, it will be a further step to make use of multiple storage devices that can respond to the required services; some being used for grid stability and others for long-term energy storage.

Within this approach further technical parameters such as system availability, reliability and losses shall also be considered. The aspects of time constraints or network capacity can be for further research. In case no adequate back-up power from storage technologies is available a reversion to fossil fuel generators is considered. By using probability curves the time-series will be run multiple times so that an accurate system can be defined.

\section{5) System analyses}

The final step performs a comparison of the energy mix of each scenario considering its additional storage. Therefore, three evaluation indicators will be considered that allow for the selection of the most appropriate scenario and energy mix for an island energy system. The three indicators are levelized cost of the overall system including the storage system, annual $\mathrm{CO}_{2}$ emissions saved and number of jobs created. Using preferences of the decision maker the most appropriate overall scenario can be determined.

Having used these compound MCDM procedures decision makers have the possibility to select the most appropriate technology mix from a very large number of technology choices. Moreover, the comparison between scenarios considering different time frames and amounts of power to be covered from RET, allows for a better understanding in defining long-term strategies for sustainable development.

\section{CONCLUSION}

Even though some of the reviewed multi-criteria decision making methods were applied in the context of islands, the amount of technologies and aspects considered was limited. Indeed, the main objectives were technology selection, site location and power optimization rather than planning for a holistic sustainable future. New concepts, including energy 
time and vector shift, are essential for island energy planning. Besides, sustainable development calls for a reduction of fossil fuels and import dependency, for which reason the usage of local abundant resources is highly encouraged. A new concept implying multiple MCDM processes to select from a vast number of technologies has be introduced.

\section{REFERENCES}

[1] E. Michalena, J. Hills, and J. P. Amat, "Developing sustainable tourism, using a multicriteria analysis on renewable energy in Mediterranean Islands," Energy for Sustainable Development, vol. 13, pp. 129-136.

[2] L. Briguglio, B. Archer, J. Jafari, and G. Wall, Sustainable Tourism in Islands and Small States: Case Studies, London: Pinter, 1996.

[3] J. Praene, M. David, F. Sinama, D. Morau, and O. Marc, "Renewable energy: Progressing towards a net zero energy island, the case of Reunion Island," Renewable and Sustainable Energy Reviews, vol. 16 , pp. 426-442, 2012

[4] J. Kelly and P. Williams, "Modelling tourism destination energy consumption and greenhouse gas emissions: Whistler, British Columbia, Canada," Journal of Sustainable Tourism, vol. 15, no. 1, pp 67-90, 2007.

[5] J. Kaldellis and K. Kavadias, "Optimal wind-hydro solution for Aegean Sea islands' electricity-demand fulfilment," Applied Energy, vol. 70, no. 4, pp. 333-354, 2001.

[6] DAFNI - Network of Aegean Islands for Sustainability, "Island sustainable energy action plan - Island of Kythnos," DAFNI - Network of Aegean Islands for Sustainability, Andros, Greece, 2012.

[7] F. Tsakiris, "Energy Development in the Non-Connected Islands of the Aegean Sea," Orkustofnun National Energy Authority, Reykjavík, 2010 .

[8] A. Andaloro, R. Salomone, L. Andaloro, N. Briguglio, and S. Sparacia, "Alternative energy scenarios for small islands: A case study from Salina Island (Aeolian Islands, Southern Italy)," Renewable Energy, vol. 47, pp. 135-146, 2012.

[9] C. Marin, "RES for paradise - Towards $100 \%$ renewable energy sources supply strategy," $100 \%$ RES - A challenge for Island Sustainable Development, Lisbon, Instituto Superior Técnico, 2005, pp. 11-15.

[10] B. Ness, E. Urbel-Piirsalu, S. Anderberg, and L. Olsson, "Categorising tools for sustainability assessment," Ecological Economics, vol. 60, pp. 498-508, 2007

[11] R. Singh, H. Murty, S. Gupta, and A. Dikshit, "An overview of sustainability assessment methodologies," Ecological Indicators, vol. 9, no. 2, pp. 189-212, 2009

[12] N. Afgan and M. Carvalho, "Multi-criteria assessment of new and renewable energy power plants," Energy, vol. 27, pp. 739-755, 2002.

[13] M. Baysal, A. Sarucan, C. Kahraman, and O. Engin, "The selection of renewable energy power plant technology using fuzzy data envelopment analysis," in Proc. the World Congress on Engineering, vol. II, London, July 2011.

[14] J. Wang, Y. Jing, C. Zhang, and J. Zhao, "Review on multi-criteria decision analysis aid in sustainable energy decision-making," Renewable and Sustainable Energy Reviews, vol. 13, no. 9, pp. 2263-2278, 2009

[15] A. Barin, L. N. Canha, A. da Rosa Abaide, and K. F. Magnago, "Selection of storage energy technologies in a power quality scenario The AHP and the fuzzy logic," in Proc. 35th Annual Conference of IEEE on Industrial Electronics, Porto, November 2009.

[16] S. Raza, I. Janajreh, and C. Ghenai, "Sustainability index approach as a selection criteria for energy storage system of an intermittent renewable energy source," in Proc. International Conference on Applied Energy ICAE 2013, Pretoria, South Africa, July 2013.

[17] A. Barin, L. N. Canha, A. Da Rosa Abaide, K. F. Magnago, B. Wottrich, and R. Q. Machado, "Multiple criteria analysis for energy storage selection," Energy and Power Engineering, vol. 3, pp. 557-564, 2011.

[18] S. Sundararagavan and E. Baker, "Evaluating energy storage technologies for wind power integration," Solar Energy, vol. 86, pp 2707-2717, 2012.

[19] Electric Power Research Institute, "Electricity energy storage technology options - A white paper primer on applications, costs, and benefits," Electric Power Research Institute, Palo Alto, California, 2010.

[20] H. Polatidis, D. Haralambopoulos, G. Munda, and R. Vreeker, "Selecting an appropriate multi-criteria decision analysis technique for renewable energy planning," Energy Sources, vol. 1, pp. 181-193, 2006.
[21] M. Beccali, M. Cellura, and M. Mistretta, "Decision-making in energy planning. Application of the Electre method at regional level for the diffusion of renewable energy technology," Renewable Energy, vol. 28, pp. 2063-2087, 2003.

[22] S. Pohekar and M. Ramachandran, "Application of multi-criteria decision making to sustainable energy planning-A review," Renewable and Sustainable Energy Reviews, vol. 8, no. 4, pp. 365-381, 2004.

[23] D. Vagiona and N. Karanikolas, "A multicriteria approach to evaluate offshore wind farms siting in Greece," Global NEST Journal, vol. 14, no. 2, pp. 235-243, 2012

[24] S. Al-Yahyaia, Y. Charabi, A. Gastli, and A. Al-Badi, "Wind farm land suitability indexing using multi-criteria analysis," Renewable Energy, vol. 44, pp. 80-87, 2012.

[25] Y. Charabi and A. Gastli, "PV site suitability analysis using GIS-based spatial fuzzy multi-criteria evaluation," Renewable Energy, vol. 36, no. 9, pp. 2554-2561, 2011.

[26] Z. Defne, K. Haas, and H. Fritz, "GIS based multi-criteria assessment of tidal stream power potential: A case study for Georgia, USA," Renewable and Sustainable Energy Reviews, vol. 15, no. 5, pp. 2310-2321, 2011.

[27] G. Hong and N. Abe, "Sustainability assessment of renewable energy projects for off-grid rural electrification: The Pangan-an Island case in the Philippines," Renewable and Sustainable Energy Reviews, vol. 16 , no. 1, pp. 54-64, 2012.

[28] D. Silva and T. Nakata, "Multi-objective assessment of rural electrification in remote areas with poverty considerations," Energy Policy, vol. 37, pp. 3096-3108, 2009.

[29] J. Cherni, I. Dyner, F. Henao, P. Jaramillo, R. Smith, and R. Font, "Energy supply for sustainable rural livelihoods. A multi-criteria decision-support system," Energy Policy, vol. 35, no. 3, pp. 1493-1504, 2007.

[30] E. Heo, J. Kim, and K. J. Boo, "Analysis of the assessment factors for renewable energy dissemination program evaluation using fuzzy AHP," Renewable and Sustainable Energy Reviews, vol. 14, pp. 2214-2220, 2010.

[31] A. Chatzimouratidis and P. Pilavachi, "Multicriteria evaluation of power plants impact on the living standard using the analytic hierarchy process," Energy Policy, vol. 36, pp. 1074-1089, 2008.

[32] F. Henao, J. Cherni, P. Jaramillo, and I. Dyner, "A multicriteria approach to sustainable energy supply for the rural poor," European Journal of Operational Research, vol. 218, no. 3, pp. 801-809, 2012.

[33] E. Stein, "A comprehensive multi-criteria model to rank electric energy production technologies," Renewable and Sustainable Energy Reviews, vol. 22, pp. 640-654, 2013.

[34] F. Ribeiro, P. Ferreira, and M. Araújo, "Evaluating future scenarios for the power generation sector using a multi-criteria decision analysis (MCDA) tool: The Portuguese case," Energy, vol. 52, pp. 126-136, 2013.

[35] F. Begic and N. Afgan, "Sustainability assessment tool for the decision making in selection of energy system-Bosnian case," Energy, vol. 32, pp. 1979-1985, 2007.

[36] M. Suo, Y. Li, and G. Huang, "Multicriteria decision making under uncertainty: An advanced ordered weighted averaging operator for planning electric power systems," Engineering Applications of Artificial Intelligence, vol. 25, no. 1, pp. 72-81, 2012.

[37] A. Papadopoulos and A. Karagiannidis, "Application of the multi-criteria analysis method Electre III for the optimisation of decentralised energy systems," Omega, vol. 36, pp. 766-776, 2008.

[38] E. L. La Rovere, J. B. Soares, L. B. Oliveira, and T. Lauria, "Sustainable expansion of electricity sector: Sustainability indicators as an instrument to support decision making," Renewable and Sustainable Energy Reviews, vol. 14, no. 1, pp. 422-429, 2010.

[39] J. Terrados, G. Almonacid, and P. Perez-Higueras, "Proposal for a combined methodology for renewable energy planning. Application to a Spanish region," Renewable and Sustainable Energy Reviews, vol. 13, pp. 2022-2030, 2009.

[40] A. Chatzimouratidis and P. Pilavachi, "Technological, economi cand sustainability evaluation of power plants using the Analytic Hierarchy Process," Energy Policy, vol. 37, pp. 778-787, 2009.

[41] K. van Alphen and W. H. M. van Sark, "Renewable energy technologies in the Maldives-determining the potential," Renewable and Sustainable Energy Reviews, vol. 11, pp. 1650-1674, 2007.

[42] C. Kahraman and I. Kaya, "A fuzzy multicriteria methodology for selection among energy alternatives," Expert Systems with Applications, vol. 37, pp. 6270-6281, 2010.

[43] S. Yi, H. Y. Sin, and E. Heo, "Selecting sustainable renewable energy source for energy assistance to North Korea," Renewable and Sustainable Energy Reviews, vol. 15, pp. 554-563, 2011.

[44] P. Blechinger and K. Shah, "A multi-criteria evaluation of policy instruments for climate change mitigation in the power generation 
sector of Trinidad and Tobago," Energy Policy, vol. 39, no. 10, pp. 6331-6343, 2011.

[45] D. Diakoulaki and F. Karangelis, "Multi-criteria decision analysis and cost-benefit analysis of alternative scenarios for the power generation sector in Greece," Renewable and Sustainable Energy Reviews, vol. 11, pp. 716-727, 2007.

[46] E. Georgopoulou, D. Lalas, and L. Papagiannakis, "A multicriteria decision aid approach for energy planning problems: The case of renewable energy option," European Journal of Operational Research, vol. 103, no. 1, pp. 38-54, 1997.

[47] T. Tsoutsos, M. Drandaki, N. Frantzeskaki, E. Iosifidis, and I. Kiosses, "Sustainable energy planning by using multi-criteria analysis application in the island of Crete," Energy Policy, vol. 37, no. 5, pp. 1587-1600, 2009.

[48] J. Burton and K. Hubacek, "Is small beautiful? A multicriteria assessment of small-scale energy technology applications in local governments," Energy Policy, vol. 35, pp. 6402-6412, 2007.

[49] F. Cavallaro and L. Ciraolo, "A multicriteria approach to evaluate wind energy plants on an Italian island," Energy Policy, vol. 33, pp. 235-244, 2005.

[50] N. Afgan and M. Carvalho, "Sustainability assessment of hydrogen energy systems," International Journal of Hydrogen Energy, vol. 29, no. 13, pp. 1327-1342, 2004.

[51] N. Afgan and M. Carvalho, "Sustainability assessment of a hybrid energy system," Energy Policy, vol. 36, pp. 2903-2910, 2008.

[52] J. Mourmouris and C. Potolias, "A multi-criteria methodology for energy planning and developing renewable energy sources at a regional level: A case study Thassos, Greece," Energy Policy, vol. 52, pp. 522-530, 2013.

[53] Y. Topcu and F. Ulengin, "Energy for the future: An integrated decision aid for the case of Turkey," Energy, vol. 29, pp. 137-154, 2004.

[54] J. Kaldellis, A. Anestis, and I. Koronaki, "Strategic planning in the electricity generation sector through the development of an integrated Delphi-based multi-criteria evaluation model," Fuel, vol. 106, pp. 212-218, 2013.

[55] T. Kaya and C. Kahraman, "Multicriteria renewable energy planning using an integrated fuzzy VIKOR \& AHP methodology: The case of Istanbul," Energy, vol. 35, pp. 2517-2527, 2010.

[56] A. Evans, V. Strezov, and T. Evans, "Assessment of sustainability indicators for renewable energy technologies," Renewable and Sustainable Energy Reviews, vol. 13, no. 5, pp. 1082-1088, 2009.

[57] N. Onat and H. Bayar, "The sustainability indicators of power production systems," Renewable and Sustainable Energy Reviews, vol. 14, no. 9, pp. 3108-3115, 2010.

[58] Varun, R. Prakash, and I. Bhat, "Energy, economics and environmental impacts of renewable energy systems," Renewable and Sustainable Energy Reviews, vol. 13, no. 9, pp. 2716-2721, 2009.

[59] F. Cavallaro, "Multi-criteria decision aid to assess concentrated solar thermal technologies," Renewable Energy, vol. 34, pp. 1678-1685, 2009.

[60] T. Buchholz, E. Rametsteiner, T. Volk, and V. Luzadis, "Multi Criteria Analysis for bioenergy systems assessments," Energy Policy, vol. 37, pp. 484-495, 2009.

[61] P. Pilavachia, S. Stephanidis, V. Pappas, and N. Afgan, "Multi-criteria evaluation of hydrogen and natural gas fuelled power plant technologies," Applied Thermal Engineering, vol. 29, pp. 2228-2234, 2009.

[62] Ö. Erol and B. Kilkis, "An energy source policy assessment using analytical hierarchy process," Energy Conversion and Management, vol. 63, pp. 245-252, 2012.

[63] K. Nigim, N. Munier, and J. Green, "Pre-feasibility MCDM tools to aid communities in prioritizing local viable renewable energy sources," Renewable Energy, vol. 29, pp. 1775-1791, 2004.

[64] D. Streimikiene, T. Balezentis, I. Krisciukaitiene, and A. Balezentis, "Prioritizing sustainable electricity production technologies: MCDM approach," Renewable and Sustainable Energy Reviews, vol. 16, pp. 3302-3311, 2012.

[65] J. San Cristobal, "Multi-criteria decision-making in the selection of a renewable energy project in spain: The Vikor method," Renewable Energy, vol. 36, pp. 498-502, 2011.

[66] P. Haurant, P. Oberti, and M. Muselli, "Multicriteria selection aiding related to photovoltaic plants on farming fields on Corsica island: A real case study using the ELECTRE outranking framework," Energy Policy, vol. 39, no. 2, pp. 676-688, 2011.
[67] L. Zhang, D. Q. Zhou, P. Zhou, and Q. T. Chen, "Modelling policy decision of sustainable energy strategies for Nanjing city: A fuzzy integral approach," Renewable Energy, vol. 62, pp. 197-203, 2014.

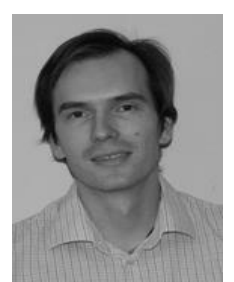

C. Wimmler graduated from the University of Applied Science Kufstein, Austria, with a BA degree in european energy business in 2010. In 2011 he received a MSc degree with distinction in renewable energy enterprise and management from Newcastle University, United Kingdom. At the moment $\mathrm{C}$. Wimmler is pursuing his $\mathrm{PhD}$ at the University of Porto, Portugal, in the sustainable energy systems MIT Portugal Program. His special fields of interest include offshore renewable energy technologies, energy planning and management as well as isolated and island energy systems.

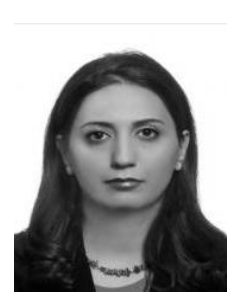

G. Hejazi was born in Tehran. She graduated from Iran University of Science and Technology with a BSc. degree in industrial engineering in 2001 and from Newcastle University with a MSc. degree in renewable energy enterprise and management in 2011. Currently she is undertaking her $\mathrm{PhD}$ degree in the sustainable energy systems MIT Portugal Program at the University of Porto, Portugal. G. Hejazi has worked for several years in the Farayaz Material Engineering Research Center and the Material and Energy Research Center. Her special fields of interest include managerial aspects of renewable energies and planning of sustainable energy systems in remote areas.

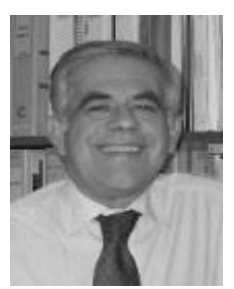

E. de Oliveira Fernandes is a full professor at the Faculty of Engineering, University of Porto, Portugal. He obtained his PhD degree on Applied Sciences at the Federal Institute of Technology (Lausanne, Switzerland) in 1973. Devoting the last 30 years of his career to teaching, research, consulting and public activities on various topics related to energy and environment, he was the founder of a RTD group on Building Thermal Physics with major pioneering activities in Portugal on passive solar technologies in buildings, indoor air quality, and energy and environment in the Urban Space.

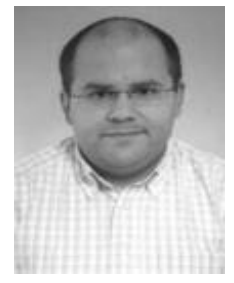

C. L. Moreira is a senior researcher in the Power Systems Unit of Instituto de Engenharia de Sistemas e Computadores do Porto (INESC Porto). He obtained the electrical engineering degree (5-year course) in 2003 and the Ph.D. degree in 2008, both from the Faculty of Engineering of Porto University, Porto, Portugal. His main research interests are focused on micro-grids dynamics and control, smart grids and smart metering.

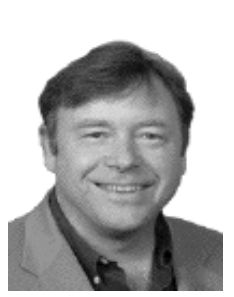

S. Connors is the director of the Analysis Group for Regional Energy Alternatives (AGREA) part of the M.I.T. Energy Initiative (MITEI). AGREA's primary research focus is in strategic planning in energy and the environment, with an emphasis on the transformation of regional energy infrastructures (e.g. "energy pathways") to simultaneously address energy security, climate change, and other energy challenges. As an extension of his role as director of AGREA, Mr Connors also coordinates several international energy initiatives involving MIT. Mr. Connors is a founding board member of the U.S. Offshore Wind Collaborative, and has served on the editorial boards of Wind Engineering and Sustainability Science. 



\section{Pollution Control}


